\title{
Anti-GITR Agonistic Monoclonal Antibody BMS-986156
}

National Cancer Institute

\section{Source}

National Cancer Institute. Anti-GITR Agonistic Monoclonal Antibody BMS-986156. NCI

Thesaurus. Code C132267.

An anti-human glucocorticoid-induced tumor necrosis factor receptor (GITR; tumor necrosis factor superfamily member 18; TNFRSF18; CD357) agonistic monoclonal antibody, with potential immune checkpoint modulating activity. Anti-GITR antibody BMS986156 binds to and activates GITR, which is expressed on the cell surface of multiple types of T-cells. This stimulates the immune system, induces both the activation and proliferation of tumor antigen-specific T-effector cells (T effs), and suppresses the function of activated T-regulatory cells (Tregs). This leads to tumor cell eradication. GITR, a member of the TNF receptor superfamily and T-cell receptor co-stimulator, is expressed on the surface of multiple immune cell types, including Tregs, Teffs, B-cells, and natural killer (NK) cells. Inappropriately activated T regs suppress both T effs and Tcell receptor (TCR) signaling. 